\title{
Synthesis and antituberculosis activity of 2-(aryl/alkylamino)-5-(4- aminophenyl)-1,3,4-thiadiazoles and their Schiff bases
}

\author{
Nilufer Solak and Sevim Rollas* \\ Marmara University, Faculty of Pharmacy, Department of Pharmaceutical Chemistry, 34668, \\ Istanbul-Turkey \\ E-mail: sevim@sevimrollas.com
}

\begin{abstract}
A series of new Schiff bases were synthesized through the condensation reaction of 1,3,4thiadiazoles containing a aromatic primary amine and 3-hydroxybenzaldehyde, salicylaldehyde, 5-nitrofurfuraldehyde or 3-nitrobenzaldehyde. The synthesized compounds screened for antituberculosis activity against Mycobacterium tuberculosis $H_{37} R v$ using BACTEC 460 radiometric system. Among the tested compounds, 2-phenylamino-5-[4-(2hydroxybenzylideneamino)phenyl]-1,3,4-thiadiazol (5a) showed the highest inhibitory activity (51\%). The activities of the synthesized Schiff bases were compared with those of the starting 2(aryl/alkylamino)-5-(4-aminophenyl)-1,3,4-thiadiazoles (4a-e). Some Schiff bases showed higher growth inhibition than the starting amines.
\end{abstract}

Keywords: Schiff bases, 1,3,4-thiadiazoles, antituberculosis activity, synthesis

\section{Introduction}

Schiff bases which contain an azomethine group attract much interest due to their synthetic along with antibacterial, ${ }^{1,2}$ antiinflammatory, ${ }^{3}$ antitumor, ${ }^{4}$ and antimycobacterial ${ }^{5}$ properties. Furthermore, thiadiazoles possess antifungal, ${ }^{6}$ antituberculosis, ${ }^{7-9}$ anticancer, ${ }^{10,11}$ antimicrobial, ${ }^{12-}$ 15 anti-inflammatory, ${ }^{16-18}$ antihypertensive, ${ }^{19,20}$ local anesthetic, ${ }^{21}$ anticonvulsant, ${ }^{22-24}$ and antitrypanosomal $^{25}$ activities.

The treatment of mycobacterial infections, especially tuberculosis, has become an important problem to solve due to the emergence of multidrug resistance. As a contribution to the new antituberculosis drug development we have previously synthesized 1,3,4-thiadiazole derivatives. ${ }^{9,} 26$ The aim of this study was to synthesize some Schiff bases having inhibition against Mycobacterium tuberculosis $H_{37} R v$ using 1,3,4-thiadiazoles, ${ }^{26}$ elucidate their structures and compare the inhibition effects of the 1,3,4-thiadiazoles and the Schiff bases. 
Tuberculosis Activity Antimicrobial Acquisition and Coordinating Facility (TAACF) of Southern Research Institute screened the compounds against Mycobacterium tuberculosis $H_{37} R v$ at $6.25 \mu \mathrm{g} / \mathrm{mL}$.

\section{Results and Discussion}

In this study, ethyl 4-(benzoylamino) benzoate (1a) was prepared by the reaction of benzocain and benzoylchloride in ether, followed by refluxing with hydrazine hydrate. ${ }^{27}$ The resulting compound was treated with different aryl/alkylisothiocyanate to prepare 1-[4(benzoylamino)benzoyl]-4-alky/arylthiosemicarbazide (3a-e), which were cyclized into 2(aryl/alkylamino)-5-(4-aminophenyl)-1,3,4-thiadiazol (4a-e) in sulfuric acid. ${ }^{26}$ Schiff bases (5a,c, 6a-d, 7a-c, 8a-c, 9a-c) were synthesized by the reaction of 1,3,4-thiadiazoles (4a-e) and 3hydroxybenzaldehyde (only 6d), salicylaldehyde, 5-nitrofurfuraldehyde or 3-nitrobenzaldehyde in methanol.

The syntheses of the Schiff bases are depicted in Scheme 1.
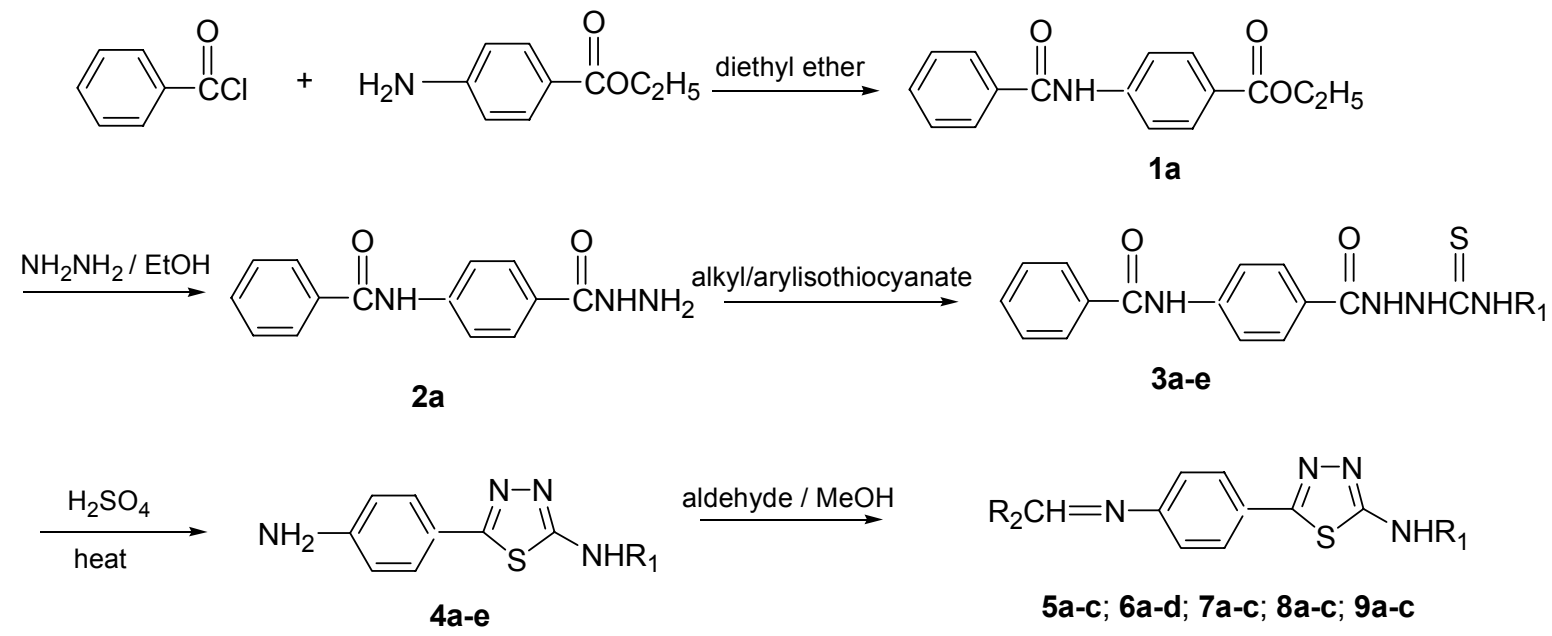

Scheme1. 5a,c: $\mathrm{R}_{1}=$ phenyl; 6a-d: $\mathrm{R}_{1}=$ benzyl; 7a-c: $\mathrm{R}_{1}=4$-methylphenyl; 8a-c: $\mathrm{R}_{1}=$ ethyl; 9a-c: $\mathrm{R}_{1}=$ methyl. 5a, 6a,7a, 8a, 9a: $\mathrm{R}_{2}=$ 2-hydroxyphenyl; 6b, 7b, 8b, 9b: $\mathrm{R}_{2}=5$-nitrofurfuryl; 5c, 6c, 7c, 8c, 9c: $\mathrm{R}_{2}=3$-nitrophenyl; 6d: $\mathrm{R}_{2}=3$-hydroxyphenyl

The presence of the imine group $(\mathrm{C}=\mathrm{N})$ in the compounds was indicated in the IR spectra between 1612-1676 $\mathrm{cm}^{-1}$. The imine group proton was observed between 10.29-8.73 ppm in the ${ }^{1} \mathrm{H}-\mathrm{NMR}$ spectra. The existence of syn-anti conformation was suggested for $7 \mathbf{a}$ and $9 \mathbf{a}$ since the imine group proton of 7a and 9a showed two singlets at 8.92, 9.03 and 9.05, 10.29 respectively. The total area under the peaks was also indicative of a single proton. In addition, the methyl protons of 7a showed chemical shifts at $2.29 \mathrm{ppm}$ and $2.38 \mathrm{ppm}$ and similarly methyl protons of 9a had two doublets at 2.93, 2.99 ppm due to syn-anti conformation. Hydroxyl protons of 5a, 6a 
and 8a synthesized from salicylaldehyde showed various resonances around 12.84-12.93 ppm due to intramolecular hydrogen bond between the hydroxyl proton and the imine group nitrogen $\left(\mathrm{OH}^{\cdots \cdots} \mathrm{N}\right) .{ }^{32}$ The ${ }^{1} \mathrm{H}-\mathrm{NMR}$ data were consistent with the literature. ${ }^{2}{ }^{33}$ Besides, in the mass spectra of compounds $5 \mathbf{a}, \mathbf{6 b}, \mathbf{6 c}, 7 \mathbf{a}, 7 \mathbf{b}, \mathbf{8 a}$ and $\mathbf{8 b}$, molecular ion peaks $\left(\mathrm{M}^{+}\right.$or $\left.\mathrm{MH}^{+}\right)$were observed at $\mathrm{m} / \mathrm{z} 373,405,416,387,406,324$ and 344 , respectively.

Table 1. Antituberculosis activity screening data of the synthesized compounds

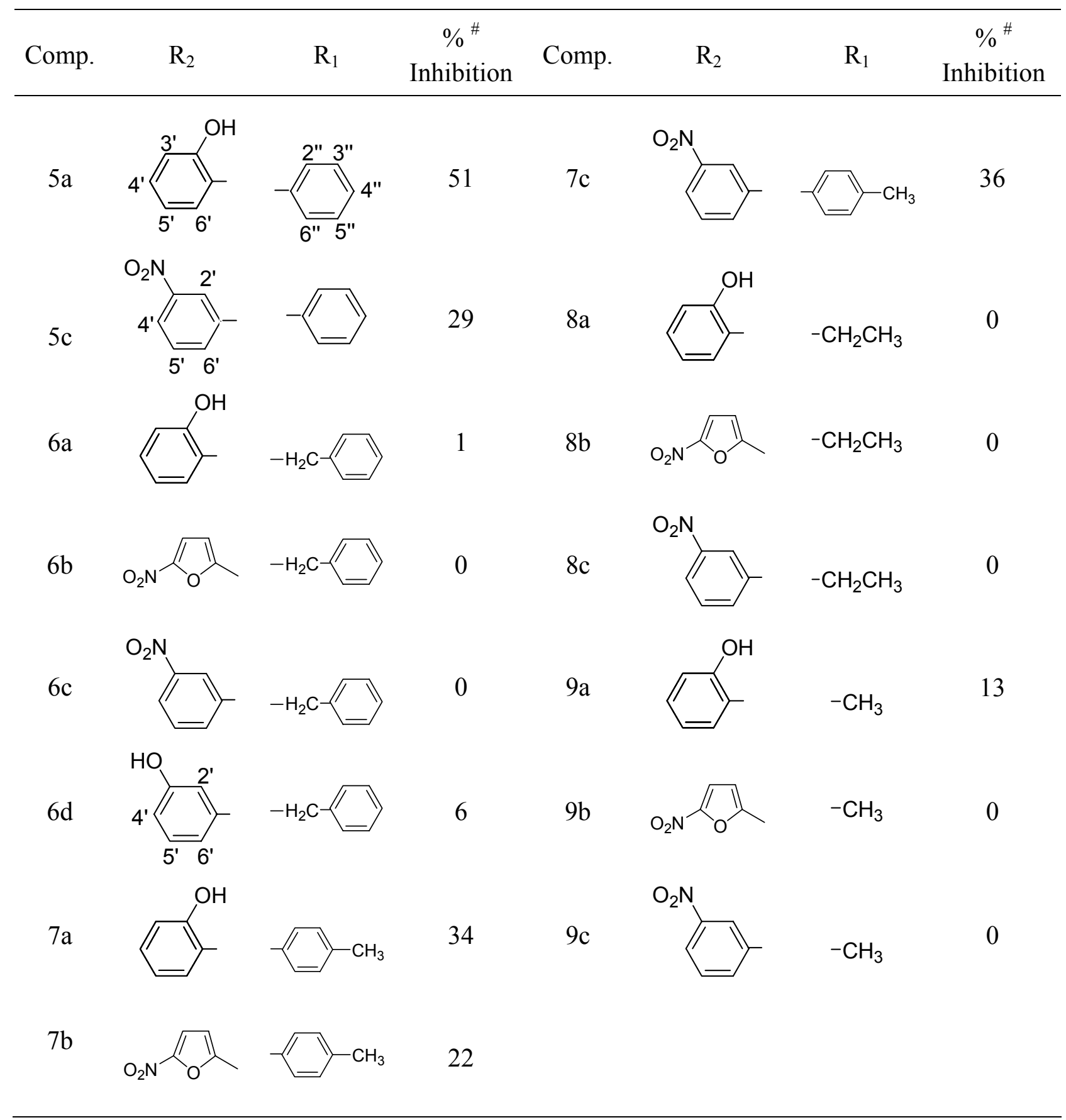

${ }^{\#} \mathrm{MIC}(\mu \mathrm{g} / \mathrm{mL})$ value $>6.25$ 
The antituberculosis tests indicated that compound 5a (containing a hydroxyl group) showed the highest inhibition (51\%) against Mycobacterium tuberculosis $H_{37} R v$ at a concentration of $>6.25 \mu \mathrm{g} / \mathrm{mL}$. Patole et al. ${ }^{5}$ indicate that conjugation of hydroxyl-rich ligands is effective in synergistically enhancing the antimycobacterial activity.

The activities of all the synthesized Schiff bases against Mycobacterium tuberculosis $H_{37} R v$ were compared with 5-(4-aminophenyl)-1,3,4-thiadiazoles (4a-e). ${ }^{26}$ The results of antituberculosis activity of the Schiff bases are given in Table 1. Compound 4a exhibited 16\% inhibition whereas compound 5a and 5c which were synthesized from $4 \mathbf{a}$ exhibited 51\%, and $34 \%$ inhibition, respectively. In addition, while $4 \mathrm{c}$ showed $22 \%$ inhibitory activity, compounds 7a, $7 \mathbf{b}$ and 7c, synthesized from $4 \mathbf{c}$, showed $34 \%, 22 \%$ and $36 \%$ inhibition, respectively.

As a result, the inhibition of the compounds, synthesized from salicylaldehyde (5a, 7a) and 3-nitrobenzaldehyde $(\mathbf{5 c}, \mathbf{7 c})$, are higher than those of $\mathbf{4 a}$ and $\mathbf{4 c}$. However, the inhibitions did not change for the compound synthesized from 5-nitrofurfuraldehyde (7b) and dramatic decreases were observed for compounds 6a-c, 8a-c and 9a-c synthesized from $\mathbf{4 b}, \mathbf{4 d}$ and $\mathbf{4 e}$, respectively. The Schiff bases (5a, 5c, 7a, 7c), synthesized through the reaction of 1,3,4thiadiazoles $(\mathbf{4 a}, \mathbf{4} \mathbf{c})$ with salicylaldehyde or 3-nitrobenzaldehyde, exhibited higher inhibitions compared to the corresponding 1,3,4-thiadiazoles $(\mathbf{4 a}, \mathbf{4 c})$. On the other hand, the inhibitions did not change for the compound $\mathbf{7 b}$ obtained by the treatment of compound $\mathbf{4 c}$ with 5nitrofurfuraldehyde. It can be concluded that although 5a is not active enough, it may be considered promising for the development of new antituberculosis agents.

\section{Experimental Section}

General Procedures. Melting points were determined on Buchi 530 and are uncorrected. The IR spectra were obtained by potassium bromide pellets using STIR-8-3000 Shimadzu spectrometer. ${ }^{1}$ H-NMR spectra were recorded on Bruker AC 200L Krotas MS-9/50 using TMS as an internal standard. The mass spectra of $\mathbf{6 b}$ and $\mathbf{8 a}$ were measured on Fisions Instruments VG Platform.II LS-MS. The mass spectra of $\mathbf{5 a}, \mathbf{6 c}, \mathbf{7 a}, \mathbf{7 b}$, and $\mathbf{8 b}$ were measured on Agilent 1100 MSD. LSMS. Elemental analysis apparatus was Leco CHNS-932. All experiments were followed by TLC using Merck Kieselgel 60 F- 0.2 mm.

Ethyl 4-(benzoylamino) benzoate (1a). Benzocain $(0.03 \mathrm{~mol})$ was dissolved in $36 \mathrm{~mL}$ ether. Benzoylchloride $(3.6 \mathrm{~mL}$ diluted in $3.6 \mathrm{~mL}$ ether) was added into the mixture drop by drop. After evaporating ether, the final mixture was washed with water until the smell of benzoyl chloride disappeared and then recrystallized from ethanol. $\mathrm{Mp} 137^{\circ} \mathrm{C} .^{27}$

4-(Benzoylamino)benzoylhydrazine (2a). $6.0 \mathrm{~mL}$ hydrazine hydrate was added to $1 \mathrm{a}(0.01$ mol). The mixture was refluxed at $110-130{ }^{\circ} \mathrm{C}$ for 45 minutes. After adding $10.0 \mathrm{~mL}$ ethanol, the mixture was heated in a water steam bath for one hour. The residue was filtered, washed with water and recrystallized from ethanol. $\mathrm{Mp} 235^{\circ} \mathrm{C} .^{27}$ 
1-(4-Benzoylamino)benzoyl-4-alky/arylthiosemicarbazide (3a-e). $80.0 \mathrm{~mL}$ ethanol was added to $2 \mathbf{a}(0.005 \mathrm{~mol})$ and heated in a water steam bath. Aryl/alkylisothiocyanate was added and refluxed for 2.5 hours. The precipitate was filtered, washed with water, recrystallized from ethanol. Mp 3a $259-260^{\circ} \mathrm{C}^{27}, 3 \mathrm{~d} 239^{\circ} \mathrm{C}^{27}, 3 \mathrm{e} 225-226^{\circ} \mathrm{C}^{27}, 3 \mathrm{~b}, 208^{\circ} \mathrm{C}^{26}, 3 \mathrm{c}, 178^{\circ} \mathrm{C}^{28}$.

2-(Aryl / alkylamino)-5-(4-aminophenyl)-1,3,4-thiadiazole (4a-e). $15.0 \mathrm{~mL} 50 \% \mathrm{H}_{2} \mathrm{SO}_{4}$ was added to 3a-e $(0.006 \mathrm{~mol})$ and refluxed at $110-150^{\circ} \mathrm{C}$ for 6 hours. The mixture was neutralized with $2 \mathrm{~N} \mathrm{NaOH}$. The precipitate was filtered, washed with water, recrystallized from ethanol. $\mathrm{Mp}$ $4 \mathrm{a} 188^{\circ} \mathrm{C}^{29}, 4 \mathrm{~b} 225^{\circ} \mathrm{C}^{26}, 4 \mathrm{c} 217-8^{\circ} \mathrm{C}^{26}, 4 \mathrm{~d} 170^{\circ} \mathrm{C}^{30}, 4 \mathrm{e} 218-220^{\circ} \mathrm{C}^{31}$.

Synthesis of Schiff bases (5a,c, 6a-d, 7a-c, 8a-c, 9a-c). 4a-e (0.001 mol) was dissolved in about $10 \mathrm{~mL}$ methanol and refluxed with aldehyde $(0.001 \mathrm{~mol})$. The precipitate was recrystallized from methanol.

2-Phenylamino-5-[4-(2-hydroxybenzylideneamino)phenyl]-1,3,4-thiadiazole

(5a) was prepared from salicylaldehyde $(0.001 \mathrm{~mol})$ and 2-(phenylamino)-5-(4-aminophenyl)-1,3,4thiadiazol 4a (0.001 mol). Yield: $41.9 \%$. Mp 258 ${ }^{\circ}$ C. Anal. Calcd. $\mathrm{C}_{21} \mathrm{H}_{16} \mathrm{~N}_{4} \mathrm{OS} .1 / 4 \mathrm{H}_{2} \mathrm{O}: \mathrm{C}$, 66.91; H, 4.27; N, 14.86. Found: C, 66.83; H, 3.41; N, 14.57. IR (KBr, cm $\left.{ }^{-1}\right) 3229(\mathrm{OH}), 3166$ $(\mathrm{NH}), 1614(\mathrm{C}=\mathrm{N}) ;{ }^{1} \mathrm{H}-\mathrm{NMR}$ (DMSO-d6) $\delta$ 6.95-7.09 (m, 2H, 3`, 5`), 7.38 (t, 2H, 4` , 6'), 7.45

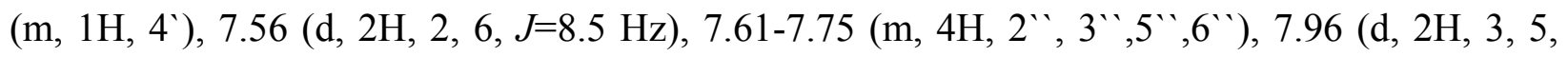
$J=8.5 \mathrm{~Hz}), 9.04(\mathrm{~s}, 1 \mathrm{H}, \mathrm{N}=\mathrm{CH}), 10.53(\mathrm{~s}, 1 \mathrm{H}, \mathrm{NH}), 12.84$ (s, 1H, OH); MS m/z: $373.1\left(\mathrm{MH}^{+}\right.$, $100 \%), 269.1(16 \%)$.

2-Phenylamino-5-[4-(3-nitrobenzylideneamino)phenyl]-1,3,4-thiadiazole (5c) was prepared from 3-nitrobezaldehyde $(0.001 \mathrm{~mol})$ and $4 \mathbf{a}(0.001 \mathrm{~mol})$. Yield: $42.6 \%$. Mp $214^{\circ} \mathrm{C}$. Anal. Calcd. $\mathrm{C}_{21} \mathrm{H}_{15} \mathrm{~N}_{5} \mathrm{O}_{2} \mathrm{~S}$ : C, 62.83; H, 3.77; N, 17.45; S, 7.99. Found: C, 62.52; H, 3.49; N, 17.11; S, 7.78. IR $\left(\mathrm{KBr}, \mathrm{cm}^{-1}\right) 3246(\mathrm{NH}), 1616(\mathrm{C}=\mathrm{N}), 1521$ (Ar-NO 2 asymmetric), 1350 (Ar- $\mathrm{NO}_{2}$ symmetric); ${ }^{1} \mathrm{H}-\mathrm{NMR}$ (DMSO-d6) $\delta 7.04$ (t, 1H, 4’), 7.38 (t, 2H, 3“', 5’'), 7.50 (d, 2H, 2, 6, J=8.4 Hz), 7.68 (d, 2H, 2 ' $\left., 6^{\prime \prime}, J=7.8 \mathrm{~Hz}\right), 7.86$ (t, 1H, 5'), 7.96 (d, 1H, 3, 5, J=8.4 Hz), 8.36-8.48 (m, 2H, 4', $\left.6^{`}\right), 8.78\left(\mathrm{~s}, 1 \mathrm{H}, 2^{`}\right), 8.91(\mathrm{~s}, 1 \mathrm{H}, \mathrm{N}=\mathrm{CH}), 10.52$ (s, 1H, NH).

2-Benzylamino-5-[4-(2-hydroxybenzylideneamino)phenyl]-1,3,4-thiadiazole (6a) was prepared from salicylaldehyde $(0.001 \mathrm{~mol})$ and 2-(benzylamino)-5-(4-aminophenyl)-1,3,4thiadiazol (4b, $0.001 \mathrm{~mol})$. Yield: $46.3 \%$. Mp 234-235 ${ }^{\circ} \mathrm{C}$. Anal. Calcd $\mathrm{C}_{22} \mathrm{H}_{18} \mathrm{~N}_{4} \mathrm{OS}$ : C, 68.37; H, 4.69; N, 14.50; S, 8.30. Found: C, 68.38; H, 4.67; N, 14.50; S, 8.18. IR ( $\left.\mathrm{KBr}, \mathrm{cm}^{-1}\right) 3212$ $(\mathrm{NH}, \mathrm{OH}), 1617(\mathrm{C}=\mathrm{N}) ;{ }^{1} \mathrm{H}-\mathrm{NMR}$ (DMSO-d6) $\delta 4.61$ (d, 2H, $\left.\mathrm{CH}_{2}, J=5.7 \mathrm{~Hz}\right), 7.03$ (t, 2H, 3', 5'),

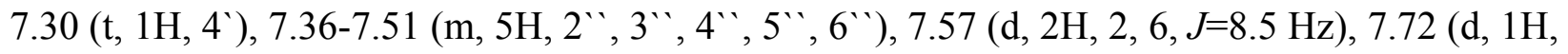
6) $7.87(\mathrm{~d}, 2 \mathrm{H}, 3,5, J=8.5 \mathrm{~Hz}), 8.49$ (t, 1H, NH), 9.05 (s, 1H, N=CH), 12.90 (s, 1H, OH).

2-Benzylamino-5-[4-(5-nitrofurfurylideneamino)phenyl]-1,3,4-thiadiazole (6b) was prepared from 5-nitrofurfuraldehyde $(0.001 \mathrm{~mol})$ and $\mathbf{4 b}(0.001 \mathrm{~mol})$. Yield: $87.6 \%$. Mp 210-211 ${ }^{\circ} \mathrm{C}$. Anal. Calcd. $\mathrm{C}_{20} \mathrm{H}_{15} \mathrm{~N}_{5} \mathrm{O}_{3} \mathrm{~S}$ : C, 59.25; H, 3.73; N, 17.27; S, 7.91. Found: C, 60.07; H, 3.25; N, 17.16; S, 7.19. IR (KBr, cm ${ }^{-1}$ ) $3245(\mathrm{NH}), 1538$ (furfural- $\mathrm{NO}_{2}$ asymmetric), 1365 (furfural- $\mathrm{NO}_{2}$ symmetric); ${ }^{1} \mathrm{H}-\mathrm{NMR}$ (DMSO-d6) $\delta 4.61$ (d, 2H, $\mathrm{CH}_{2}, J=5.8 \mathrm{~Hz}$ ), 7.09-7.69 (m, 8H, 2, 2 ", 3" , 4', 4”, 5’ , 6, 6“'), 7.80-7.99 (m, 3H, 3, 3`, 5), 8.52 (t, 1H, NH), 8.74 (s, 1H, N=CH). MS m/z: $405\left(\mathrm{M}^{+}, 6.6 \%\right), 91(100.0 \%)$. 
2-Benzylamino-5-[4-(3-nitrobenzylideneamino)phenyl]-1,3,4-thiadiazole (6c) was prepared from 3-nitrobenzaldehyde $(0.001 \mathrm{~mol})$ and $\mathbf{4 b}(0.001 \mathrm{~mol})$. Yield: $45.2 \%$. Mp 207-208 ${ }^{\circ} \mathrm{C}$. Anal. Calcd. $\mathrm{C}_{22} \mathrm{H}_{17} \mathrm{~N}_{5} \mathrm{O}_{2} \mathrm{~S}$ : C, 63.60; H, 4.12; N, 16.86; S, 7.72. Found: C, 64.49; H, 3.60; N, 16.71; S, 6.83. IR $\left(\mathrm{KBr}, \mathrm{cm}^{-1}\right) 3333(\mathrm{NH}), 1633(\mathrm{C}=\mathrm{N}), 1524\left(\mathrm{Ar}-\mathrm{NO}_{2}\right.$ asymmetric), $1352\left(\right.$ Ar- $\mathrm{NO}_{2}$ symmetric); ${ }^{1} \mathrm{H}-\mathrm{NMR}$ (DMSO-d6) $\delta 4.56\left(\mathrm{~d}, 2 \mathrm{H}, \mathrm{CH}_{2}\right), 7.29\left(\mathrm{t}, 1 \mathrm{H}, 4{ }^{\prime}, J=5.8 \mathrm{~Hz}\right), 7.36-7.54$ (m, $\left.6 \mathrm{H}, 2,2^{\prime \prime}, 3^{\prime \prime}, 5^{\prime \prime}, 6,6^{\prime \prime}\right), 7.78\left(\mathrm{~m}, 3 \mathrm{H}, 3,5,5^{`}\right), 8.42$ (d, 2H, 4', 6', J=8.1 Hz), 8.48 (t, 1H, NH), 8.80 (s, 1H, 2'), 8.92 (s, 1H, N=CH). MS m/z: 416.2. ( $\left.\mathrm{MH}^{+}, 100.0 \%\right), 91.1(23.6 \%)$.

2-Benzylamino-5-[4-(3-hydroxybenzylideneamino)phenyl]-1,3,4-thiadiazole (6d) was prepared from 3-hydroxybenzaldehyde $(0.001 \mathrm{~mol})$ and $\mathbf{4 b}(0.001 \mathrm{~mol})$. Yield: $53.2 \%$. Mp $230^{\circ}$ C. Anal. Calcd. $\mathrm{C}_{22} \mathrm{H}_{18} \mathrm{~N}_{4} \mathrm{OS}$ : C, 68.37; H, 4.69; N, 14.50; S, 8.30. Found: C, 68.45; H, 4.34; $\mathrm{N}, 14.15$; S, 7.73. IR (KBr, cm $\left.{ }^{-1}\right) 3395(\mathrm{NH}, \mathrm{OH}), 1624(\mathrm{C}=\mathrm{N}) ;{ }^{1} \mathrm{H}-\mathrm{NMR}(\mathrm{DMSO}-\mathrm{d} 6) \delta 4.59$ (d,

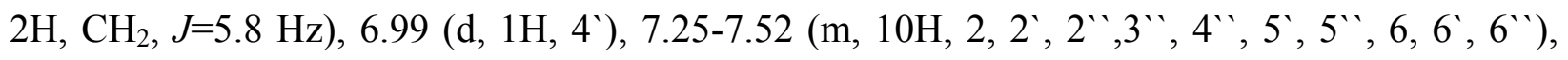
$7.81(\mathrm{~d}, 2 \mathrm{H}, 3,5, J=8.4 \mathrm{~Hz}), 8.46(\mathrm{t}, 1 \mathrm{H}, \mathrm{NH}), 8.62(\mathrm{~s}, 1 \mathrm{H}, \mathrm{N}=\mathrm{CH}), 9.71(\mathrm{~s}, 1 \mathrm{H}, \mathrm{OH})$;

2-(4-Methylphenylamino)-5-[4-(2-hydroxybenzylideneamino)phenyl]-1,3,4-thiadiazole (7a) was prepared from salicylaldehyde $(0.001 \mathrm{~mol})$ and 2-(4-metylphenylamino)-5-(4-aminophenyl)1,3,4-thiadiazol (4c, $0.001 \mathrm{~mol}$ ). Yield: $72.1 \%$. Mp 254-255 ${ }^{\circ} \mathrm{C}$. Anal. Calcd. $\mathrm{C}_{22} \mathrm{H}_{18} \mathrm{~N}_{4} \mathrm{OS}: \mathrm{C}$, 68.37; H, 4.69; N, 14.50; S, 8.30. Found: C, 68.36; H, 4.28; N, 14.45; S, 8.32. IR (KBr, cm $\left.{ }^{-1}\right)$ $3433(\mathrm{NH}, \mathrm{OH}), 1617(\mathrm{C}=\mathrm{N})$ ) ${ }^{1} \mathrm{H}-\mathrm{NMR}$ (DMSO-d6) $\delta 2.29$ and 2.38 (two s, 3H, $\mathrm{CH}_{3}$ ), 6.89-7.98

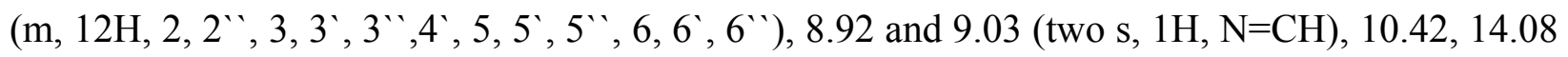
(two s, $1 \mathrm{H}, \mathrm{OH}) ; 12.52$ and 12.98 (two s, $1 \mathrm{H}, \mathrm{NH})$; MS m/z: $387.2\left(\mathrm{MH}^{+}, 100.0 \%\right.$ ), 186 (10.9 $\%)$.

2-(4-Methylphenylamino)-5-[4-(5-nitrofurfurylideneamino)phenyl]-1,3,4-thiadiazole (7b) was prepared from 5-nitrofurfuraldehyde $(0.001 \mathrm{~mol})$ and $4 \mathbf{c}(0.001 \mathrm{~mol})$. Yield: $54.3 \%$. Mp 224-225 ${ }^{\circ}$. Anal. Calcd. $\mathrm{C}_{20} \mathrm{H}_{15} \mathrm{~N}_{5} \mathrm{O}_{3} \mathrm{~S}$ : C, 59.25; H, 3.72; S, 7.90. Found: C, 59.12; H, 3.44; S, 7.38. IR $\left(\mathrm{KBr}, \mathrm{cm}^{-1}\right) 3387(\mathrm{NH}), 1616(\mathrm{C}=\mathrm{N}), 1538$ (furfural- $\mathrm{NO}_{2}$ asymmetric), 1352 (furfural$\mathrm{NO}_{2}$ symmetric); ${ }^{1} \mathrm{H}-\mathrm{NMR}$ (DMSO-d6) $\delta 2.32$ (s, 3H, $\left.\mathrm{CH}_{3}\right) ; 7.21$ (d, 2H, 2 " , 6 ", J=8.4 Hz);

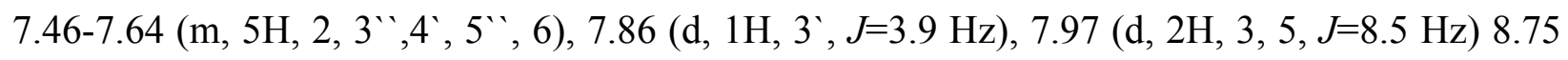
(s, 1H, N=CH), 10.46 (s, 1H, NH ); MS m/z: $406.1\left(\mathrm{MH}^{+}, 100.0 \%\right), 282(10.1 \%)$.

2-(4-Methylphenylamino)-5-[4-(3-nitrobenzylideneamino)phenyl]-1,3,4-thiadiazole (7c) was prepared from 3-nitrobezaldehyde $(0.001 \mathrm{~mol})$ and $4 \mathbf{c}(0.001 \mathrm{~mol})$. Yield: $41.2 \%$. Mp $255^{\circ} \mathrm{C}$. Anal. Calcd. $\mathrm{C}_{22} \mathrm{H}_{17} \mathrm{~N}_{5} \mathrm{O}_{2} \mathrm{~S}$ : C, 63.60; H, 4.12; N, 16.86; S, 7.72. Found: C, 63.87; H, 3.95; N, 16.86; S, 7.21. IR (KBr, cm $\left.{ }^{-1}\right) 3382(\mathrm{NH}), 1612(\mathrm{C}=\mathrm{N}), 1517$ (Ar- $\mathrm{NO}_{2}$ asymmetric), 1350 (Ar$\mathrm{NO}_{2}$ symmetric); ${ }^{1} \mathrm{H}-\mathrm{NMR}$ (DMSO-d6) $\delta 2.27$ (s, 3H, $\left.\mathrm{CH}_{3}\right), 7.21$ (d, 2H, $\left.{ }^{\prime}{ }^{\prime}, 6^{\prime \prime}, J=8.4 \mathrm{~Hz}\right), 7.51$ $(\mathrm{d}, 2 \mathrm{H}, 2,6, J=8.5 \mathrm{~Hz}), 7.58$ (d, 2H, 3“ '5 ',$J=8.4 \mathrm{~Hz}), 7.88$ (t, 1H, 5 ), 7.98 (d, 2H, 3, 5, J=8.5 $\mathrm{Hz}), 8.38-8.49$ (m, 2H, 4',6'), 8.81 (s, 1H, 2`), 8.93 (s,1H, N=CH), 10.44 (s, 1H, NH).

2-Ethylamino-5-[4-(2-hydroxybenzylideneamino)phenyl]-1,3,4-thiadiazole (8a) was prepared from salicylaldehyde $(0.001 \mathrm{~mol})$ and 2-ethylamino-5-(4-aminophenyl)-1,3,4-thiadiazol (4d, 0.001 mol). Yield: $82.3 \%$. Mp $216^{\circ} \mathrm{C}$. Anal. Calcd. $\mathrm{C}_{17} \mathrm{H}_{16} \mathrm{~N}_{4} \mathrm{OS}: \mathrm{C}, 62.94 ; \mathrm{H}, 4.97 ; \mathrm{N}, 17.27$; S, 9.88. Found: C, 63.41; H, 5.70; N, 17.40; S, 9.40. IR ( KBr, cm $\left.{ }^{-1}\right) 3353(\mathrm{NH}, \mathrm{OH}), 1617(\mathrm{C}=\mathrm{N})$; ${ }^{1} \mathrm{H}-\mathrm{NMR}(\mathrm{DMSO}-\mathrm{d} 6) \delta 1.27\left(\mathrm{t}, 3 \mathrm{H}, \mathrm{CH}_{3}\right), 3.39-3.43\left(\mathrm{~m}, 2 \mathrm{H}, \mathrm{CH}_{2}\right), 6.98-7.12\left(\mathrm{~m}, 2 \mathrm{H}, 3^{`}, 5^{`}\right)$, 
7.42-7.63 (m, 3H, 2, 4', 6), $7.74(\mathrm{~m}, 1 \mathrm{H}, 6), 7.87$ (d, 2H, 3, 5, J=8.6 Hz), 7.98 (t, 1H, NH), 9.07 $(\mathrm{s}, 1 \mathrm{H}, \mathrm{N}=\mathrm{CH}), 12.93(\mathrm{~s}, 1 \mathrm{H}, \mathrm{OH}) ; \mathrm{MS} m / z: 324\left(\mathrm{M}^{+}, 65.6 \%\right), 296,221,120(78.3 \%), 102$ $(67.4 \%), 60(100 \%), 41(86.4 \%)$.

2-Ethylamino-5-[4-(5-nitrofurfurylideneamino)phenyl]-1,3,4-thiadiazole (8b) was prepared from 5-nitrofurfuraldehyde $(0.001 \mathrm{~mol})$ and $4 \mathbf{d}(0.001 \mathrm{~mol})$. Yield: $87.4 \%$. Mp $220^{\circ} \mathrm{C}$. Anal. Calcd. $\mathrm{C}_{15} \mathrm{H}_{13} \mathrm{~N}_{5} \mathrm{O}_{3} \mathrm{~S} . \mathrm{H}_{2} \mathrm{O}$ : C, 49.86; H, 4.15; S, 8.86. Found: C, 50.63; H, 3.86; S, 8.46. IR (KBr, $\left.\mathrm{cm}^{-1}\right) 3251(\mathrm{NH}), 1655(\mathrm{C}=\mathrm{N}), 1549\left(\right.$ Ar- $\mathrm{NO}_{2}$ asymmetric), 1354 (Ar-NO $\mathrm{N}_{2}$ symmetric); ${ }^{1} \mathrm{H}-\mathrm{NMR}$ (DMSO-d6) $\delta 1.25$ (t, 3H, $\mathrm{CH}_{3}$ ), 3.38-3.55 (m, 2H, $\mathrm{CH}_{2}$ ), 7.46-7.55 (m, 3H, 2, 4 , 6), 7.81-7.92 (m, 3H, 3, 3`, 5), 7.97 (t, 1H, NH), $8.73(\mathrm{~s}, 1 \mathrm{H}, \mathrm{N}=\mathrm{CH})$; MS m/z: $344.1\left(\mathrm{MH}^{+}, 100.0 \%\right), 220.1$ $(9.2 \%)$.

2-Ethylamino-5-[4-(3-nitrobenzylideneamino)phenyl]-1,3,4-thiadiazole (8c) was prepared from 3-nitrobenzaldehyde $(0.001 \mathrm{~mol})$ and $4 \mathbf{d}(0.001 \mathrm{~mol})$. Yield: $58.2 \%$. Mp 205-206 ${ }^{\circ} \mathrm{C}$. Anal. Calcd. $\mathrm{C}_{17} \mathrm{H}_{15} \mathrm{~N}_{5} \mathrm{O}_{2} \mathrm{~S}$ : C, 57.78; H, 4.28; N, 19.82; S, 9.07. Found: C, 57.37; H, 3.77; N, 19.55; S, 9.07. IR ( $\left.\mathrm{KBr}, \mathrm{cm}^{-1}\right) 3200(\mathrm{NH}), 1635(\mathrm{C}=\mathrm{N}), 1533\left(\right.$ Ar- $\mathrm{NO}_{2}$ asymmetric), $1353\left(\mathrm{Ar}-\mathrm{NO}_{2}\right.$ symmetric); ${ }^{1} \mathrm{H}-\mathrm{NMR}$ (DMSO-d6) $\delta 1.27$ (t, 3H, $\left.\mathrm{CH}_{3}\right), 3.32-3.48\left(\mathrm{~m}, 2 \mathrm{H}, \mathrm{CH}_{2}\right), 7.32$ (d, 2H, 2, 6, $J=8.3 \mathrm{~Hz}$ ), 7.72 (t, 1H, NH), 7.79 (d, 3H, 3, 5, 5'), 8.29 (t, 2H, 4', 6'), 8.69 (s, 1H, $\left.2^{`}\right), 8.76$ (s, $1 \mathrm{H}, \mathrm{N}=\mathrm{CH}$ ).

2-Methylamino-5-[4-(2-hydroxybenzylideneamino)phenyl]-1,3,4-thiadiazole (9a) was prepared from salicylaldehyde $(0.001 \mathrm{~mol})$ and 2-(methylamino)-5-(4-aminophenyl)-1,3,4thiadiazol (4e, $0.001 \mathrm{~mol})$. Yield: $71.9 \%$. Mp 204-205 ${ }^{\circ} \mathrm{C}$. Anal. Calcd. $\mathrm{C}_{16} \mathrm{H}_{14} \mathrm{~N}_{4} \mathrm{OS}$ : C, 61.92; H, 4.55; N, 18.05; S, 10.33. Found: C, 61.99; H, 4.57; N, 18.02; S, 10.40. IR (KBr, cm ${ }^{-1)} 3180$ $(\mathrm{NH}, \mathrm{OH}), 1615(\mathrm{C}=\mathrm{N})$; ${ }^{1} \mathrm{H}-\mathrm{NMR}$ (DMSO-d6) 2.93 and 2.99 (two d, 3H, $\mathrm{CH}_{3}, J=4.7 \mathrm{~Hz}, 4.7$ $\mathrm{Hz}$ ); 5.54 and 6.65 (s and d, 1H, NH); 6.91-7.18 (m, 2H, 3', 5'); 7.40-7.52 (m, 1H, $\left.4^{\prime}\right)$; 7.55 (d, $2 \mathrm{H}, 2,6, J=8.5 \mathrm{~Hz}$ ); 7.72 (d, 1H, 6', $=7.6 \mathrm{~Hz}$ ); 7.91 (d, 2H, 3, 5, J=8.5 Hz); 9.05 and 10.29 (two s, $1 \mathrm{H}, \mathrm{N}=\mathrm{CH}$ ); 10.71 and 12.91 (two s, $1 \mathrm{H}, \mathrm{OH}$ ).

2-Methylamino-5-[4-(5-nitrofurfurylideneamino)phenyl]-1,3,4-thiadiazole (9b) was prepared from 5-nitrofurfuraldehyde $(0.001 \mathrm{~mol})$ and $4 \mathrm{e}(0.001 \mathrm{~mol})$. Yield: $62.7 \%$. Mp 210-211 ${ }^{\circ} \mathrm{C}$. Anal. Calcd. $\mathrm{C}_{14} \mathrm{H}_{11} \mathrm{~N}_{5} \mathrm{O}_{3} \mathrm{~S}$ : C, 51.06; H, 3.37; N, 21.27; S, 9.74. Found: C, 51.66; H, 3.54; N, 21.34; S, 9.90. IR $\left(\mathrm{KBr}, \mathrm{cm}^{-1}\right) 3350(\mathrm{NH}), 1624(\mathrm{C}=\mathrm{N}), 1534\left(\mathrm{Ar}-\mathrm{NO}_{2}\right.$ asymmetric and N-H), 1347 (Ar$\mathrm{NO}_{2}$ symmetric); ${ }^{1} \mathrm{H}-\mathrm{NMR}$ (DMSO-d6) $\delta 2.73$ (d, 3H, $\mathrm{CH}_{3}, J=4.8 \mathrm{~Hz}$ ); 7.17-7.34 (m, 3H, 2, 4', 6); 7.52-7.75 (m, 4H, NH, 3, 3`, 5), 8.47 (s, 1H, N=CH).

2-Methylamino-5-[4-(3-nitrobenzylideneamino)phenyl]-1,3,4-thiadiazole (9c) was prepared from 3-nitrobenzaldehyde $(0.001 \mathrm{~mol})$ and $4 \mathrm{e}(0.001 \mathrm{~mol})$. Yield: $75.1 \%$. Mp $226^{\circ} \mathrm{C}$. Anal. Calcd. $\mathrm{C}_{16} \mathrm{H}_{13} \mathrm{~N}_{5} \mathrm{O}_{2} \mathrm{~S}$ : C, 56.63; H, 3.86; N, 20.64; S, 9.45. Found: C, 56.32; H, 3.55; N, 20.18; S, 9.19. IR ( $\left.\mathrm{KBr}, \mathrm{cm}^{-1}\right) 3324(\mathrm{NH}), 1624(\mathrm{C}=\mathrm{N}), 1525\left(\mathrm{Ar}-\mathrm{NO}_{2}\right.$ asymmetric), $1351\left(\mathrm{Ar}-\mathrm{NO}_{2}\right.$ symmetric); ${ }^{1} \mathrm{H}-\mathrm{NMR}$ (DMSO-d6) $\delta 3.08$ (d, 3H, $\left.\mathrm{CH}_{3}, J=4.8 \mathrm{~Hz}\right), 7.58$ (d, 2H, 2, 6, J=8.5 Hz), 7.93-8.03 (m, 4H, NH, 3, 5, 5`), 8.53 (d, 2H, 4', 6' J=8.4 Hz), 8.89 (s, 1H, 2`), 9.02 (s, 1H, $\mathrm{N}=\mathrm{CH})$.

Antituberculosis activity assays. Tuberculosis Activity Antimicrobial Acquisition and Coordinating Facility (TAACF) of Southern Research evaluated all of the compounds for in vitro 
antituberculosis activity against mycobacterium tuberculosis $H 37 R v$. Primary screening was conducted at $6.25 \mu \mathrm{g} / \mathrm{mL}$ against mycobacterium tuberculosis H37Rv in BACTEC 12B medium using broth microdilution assay ${ }^{34}$

\section{Acknowledgements}

We thank Dr Joseph A. Maddry from the Tuberculosis Antimicrobial Acquisition and Coordination Facility (TAACF) for the in vitro evaluation of antimycobacterial activity using Mycobacterium tuberculosis $H_{37} R v$. The mass analysis was supported by Marmara University Scientific Research Project Commission, Project number: SAG-YYP-151105-0221.

\section{References}

1. Abd El Rahman, A. H.; Ismail E. M. Arzneim.-Forsch/Drug Res. 1976, 26, 756.

2. Dogan, H. N.; Buyuktimkin, S.; Rollas, S.; Yemni, E.; Cevikbas, A. Farmaco 1997, 52, 565.

3. Atta, S. M. Sh.; Ammen, A. Arzneim.-Forsch./Drug Res. 1993, 43,1354.

4. Dhapalapur, M. G.; Sabnis, S. S.; Deliwala, C. V. J. Med. Chem. 1968, 11, 1014.

5. Patole J.; Shingnapurkar D.; Padhye S.; Ratledge C. Bioorg. Med. Chem. Lett. 2006, 16, 1514.

6. Foroumadi, F.; Daneshtalab, M.; Shafiee, A. Arzneim.-Forsch./Drug Res.1999, 49, 1035.

7. Foroumadi, F.; Mirzaei, M.; Shafiee, A. Farmaco 2001, 56, 621.

8. Karakus, S.; Rollas, S. Farmaco 2002, 57, 577.

9. Oruc, E. E.; Rollas, S.; Kandemirli, F.; Shvets, N.; Dimoglo, A. S. J. Med. Chem. 2004, 47, 6760 .

10. Miyamoto, K.; Koshiura, R.; Moro, M.; Yokoi, H.; Mori, C.; Hasegawa, T.; Takatori, K. Chem. Pharm. Bull. 1985, 33, 5126.

11. Chou, J. Y.; Lai, S. Y.; Pan, S. L.; Jow, G. M.; Chern, J. W.; Guh, J. H. Biochem Pharmacol. 2003, 66, 115.

12. Desai, K.; Baxi, A. J. Indian J. Pharm. Sci. 1992, 54, 183.

13. Gawande, N. G.; Shingare, M. S. Indian J. Chem. 1987, 26B, 387.

14. Mamolo, M. G.; Vio, L.; Banfi, E. Farmaco 1996, 51, 71.

15. Demirbas N.; Demirbas A. ; Karaoglu S.A. ; Celik E. Arkivoc 2005, (i), 75.

16. Mullican, M. D.; Wilson, M. W.; Connor, D. T.; Kostlan, C. R.; Schrier, D. J.; Dyer, R.D. J. Med. Chem. 1993, 36, 1090.

17. Song, Y.; Connor, D. T.; Sercel, A. D.; Sorenson, R. J.; Doubleday, R.; Unangst, P. C.; Roth, B. D.; Beylin, V. G.; Gilbertsen, R. B.; Chan, K.; Schrier, D.J.; Guglietta, A.; Bornemeier, D. A.; Dyer, R. D. J. Med. Chem. 1999, 42, 1161. 
18. Labanauskas, L.; Kalcas, V.; Udrenaite, E.; Gaidelis, P.; Brukstus, A.; Dauksas, A. Pharmazie 2001, 56, 617.

19. Turner, S.; Myers, M., Gadie, B.; Nelson, A. J.; Pape, R.; Saville, J. F.; Doxey, J. C.; Berridge, T. L. J. Med. Chem. 1988, 31, 902.

20. Turner, S.; Myers, M., Gadie, B.; Hale, S. A.; Horsley, A.; Nelson, A. J.; Pape, R.; Saville, J. F.; Doxey, J.C.; Berridge, T.L. J. Med. Chem. 1988, 31, 907.

21. Mazzone, G.; Pignatello, R.; Mazzone, S.; Panico, A.; Penisi, G.; Castana, R.; Mazzone, P. Farmaco 1993, 48, 1207.

22. Chapleo, C. B.; Myers, M.; Myers, P. L.; Saville, J. F.; Smith, A. C. B.; Stillings, M. R.; Tulloch, I. F.; Walter, D. S.; Welbourn, A. J. Med. Chem. 1986, 29, 2273.

23. Chapleo, C. B.; Myers, P. L.; Smith, A. C. B.; Stillings, M. R.; Tulloch, I. F.; Walter, D. S. J. Med. Chem. 1988, 31, 7.

24. Dogan, H. N.; Duran, A.; Rollas, S.; Sener, G.; Uysal, M. K.; Gulen, D. Bioorg. Med. Chem. 2002, 10, 2893.

25. Carvalho, S.; Silva E.F.; Santa-Rita R.M.; Castro S.L.; Fraga C.A.M. Bioorg. Med. Chem. Lett. 2004, 14, 5967.

26. Karakus, S. Ph.D. thesis, Marmara University, Institution of Healt Sciences 2001.

27. Kalyoncuoglu, N.; Rollas, S.; Sur-Altıner, D.; Yegenoglu, Y.; Ang, O. Pharmazie 1992, 47, 796.

28. Kucukguzel, I..; Kucukguzel, S.G.; Rollas, S.; Otuk-Sanis, G.; Ozdemir, O.; Bayrak, I., Altug, T.; Stables, J. P. Il Farmaco 2004, 59,893.

29. Rollas, S. J. Pharm. Univ. Mar. 1985,1, 59.

30. Rollas, S.; Topaloglu , Y, J. Pharm.Univ. Mar. 1986, 2, 1.

31. Ozger, Y.; Rollas, S. Mar. Univ. J. Sci. and Tech. 1998, 5, 133.

32. Szady-Chelmieniecka, A.; Grech, E.; Rozwadowski, Z.; Dziembowska, T.; Schilf, W.; Kamienski, B. Journal of Molecular Structure 2001, 565-566, 125.

33. Terzioglu, N.; Karali, N.; Gursoy, A.; Otuk, G.; Kiraz, M.; Erturan, Z. Acta Pharm. Turcia, 1998, 40, 77.

34. (a) Collins, L. ; Franzblau, S. G. Antimicrob. Agents Chemother. 1997, 1004. (b) TAACF web site www.taacf.org 